\title{
Non-Vacuum LRS Bianchi Type-V Cosmological Model in $f(R)$ Theory of Gravity
}

\author{
L.S. Ladke ${ }^{1}$ \\ R. A. Hiwarkar ${ }^{2}$ \\ Department of Mathematics \\ Nilkanthrao Shinde Science and Arts college \\ Bhandravati, India \\ lemrajvandana@gmail.com \\ V. K. Jaiswal ${ }^{3}$ \\ Department of Mathematics \\ Guru Nanak Institute of Engg. \& Technology \\ Nagpur, India \\ rhiwarkar@gmail.com \\ Department of Mathematics \\ Priyadarshini J. L. College of Engineering, \\ Nagpur, India \\ vkjassi@gmail.com
}

\begin{abstract}
We study the non-vacuum perfect fluid solution of LRS Bianchi type-V space-time in the $f(R)$ theory of gravity to obtained energy density and pressure of the universe by using stiff matter. The time dependent deceleration parameter is used to discuss the physical behavior of the model. The function of the Ricci scalar is also evaluated.
\end{abstract}

Keywords: LRS Bianchi type-V space-time, $f(R)$ theory of gravity, deceleration parameter.

\section{INTRODUCTION}

The general theory of relativity has the tremendous success which explains the most of gravitational phenomena, but it fails to explain the nature of the negative component known as dark energy and accelerating expansion of the universe. Therefore there is a need of modification to general theory of relativity. Recent observations also strongly support that the universe is an accelerated state which is conformed from CMB [1-6]. Plenty of modifications had been done. Among them each one has its novel features. The $f(R)$ theory of gravity is one of the modified theory which answered the problem of dark matter, dark energy and accelerating expansion of the universe. This theory has such important characteristic, hence it considered as most suitable.

Many authors explained the concept of $f(R)$ theory of gravity. The late time acceleration and early time deceleration confirmed by Nijiri and Odinstov [7] in $f(R)$ theory of gravity. The Nojiri and Odintsov [8] have studied various modified gravity theories that are considered as gravitational alternatives for dark energy. M. Sharif and H. Rizwana Kausar [9] explained anisotropic Bianchi typeIII model in $f(R)$ gravity by assuming exponential and power-law volumetric expansion which represent an accelerated expansion of the universe. M. Sharif et. al. [10] explained the energy distribution in $f(R)$ gravity by using generalized Landau-Lifshitz energy-momentum complex. K. S. Adhav [11] obtained Bianchi type-III string cosmological model in $f(R)$ gravity. Hollenstein and Francisco S. N. Lobo [12] discussed the exact solutions of $f(R)$ gravity coupled to nonlinear electrodynamics. Many authors have done wonderful work on $f(R)$ theory of gravity in different context.

The investigation of Bianchi type models in modified $f(R)$ theory of gravity is an interesting discussion. The anomalies found in the cosmic microwave background (CMB) and large scale structure observations stimulated a growing interest in anisotropic cosmological models of the universe. Kumar and Singh [13] solved Bianchi type-I space-time in general relativity in the presence of perfect fluid. M. Sharif and H. Rizwana Kausar [14] discussed non-vacuum solutions of Bianchi 
Type- $\mathrm{VI}_{0}$ universe in $f(R)$ gravity considering isotropic perfect fluid. M. Sharif and M. Farasat Shamir [15] exhibited perfect fluid solutions of Bianchi types-I and V space-times in $f(R)$ theory of gravity. The Bianchi models being anisotropic are useful to study isotropic behavior of the universe with the passage of time. Sharif and Kausar [16] studied the isotropic behavior of Bianchi III model in $f(R)$ gravity. The scalar field can play a vital role to explain the cosmic acceleration which has widely been studied in $f(R)$ gravity [17,18]. As $f(R, T)$ theory involves coupling between matter and geometry, so considering scalar field as a source may provide some new insights. Farasat Shamir et. al.[19] obtained exact solutions of Bianchi types-I \&V models in $f(R, T)$ gravity which gives constant declaration parameter by using variation law of Hubble parameter. R.L.Naidu et. al.[20] discussed FRW viscous fluid cosmological model in $f(R, T)$ gravity. H.R. Ghate et. al.[21] studied Bianchi type-IX viscous string cosmological model in $f(R, T)$ gravity with special form of declaration parameter. P.K. Sahoo et. al. [22] investigated Kaluza-Klein cosmological model in $f(R, T)$ gravity with $\Lambda(t)$ by considering constant decelerating parameter.

Bianchi models play a significant role because these models are homogeneous and anisotropic in nature, in which the process of isotropization of the universe is studied through the passage of time. The study of Bianchi type- $\mathrm{V}$ cosmological model create more interest because these models are anisotropic generalization of open FRW model and allow arbitrarily small anisotropy levels at any constant of cosmic time. Bianchi type-V model have been studied in details by number of authors viz. Banerjee and Sanyal [23], Collins [24], Wainwrigh et al. [25].

We are attentive in the $f(R)$ theory of gravity. With such motivation, in this paper, we have examined non-vacuum perfect fluid solution of LRS Bianchi type-V space-time in the $f(R)$ theory of gravity. We used stiff matter to obtained energy density and pressure of the universe. Also we used time dependent deceleration parameter to discuss the physical behavior of the model. The function of the Ricci scalar is also evaluated.

\section{Field Equations in $f(R)$ Theory of Gravity}

The $f(R)$ theory of gravity is the simplest generalization of the general theory of relativity proposed by Einstein in which Ricci scalar in Einstein-Hilbert action is replaced by an arbitrary function of the Ricci scalar. The field equations in $f(R)$ theory of gravity are given by

$$
F(R) R_{i j}-\frac{1}{2} f(R) g_{i j}-\nabla_{i} \nabla_{j} F(R)+g_{i j} \square F(R)=k T_{i j},
$$

where $F(R) \equiv \frac{d f(R)}{d R}, \square \equiv \nabla^{i} \nabla_{i}, \nabla_{i}$ is the covariant derivative and $T_{i j}$ is the standard matter energy momentum tensor.

If we take $f(R)=R$, the field equations (1) reduce to the field equation of general theory of relativity which is proposed by Einstein.

\section{LRS BIANCHI TYPE-V MODEL}

The line element of LRS Bianchi type- $\mathrm{V}$ space-time is given by

$$
d s^{2}=d t^{2}-A^{2} d x^{2}-e^{2 m x} B^{2}\left[d y^{2}+d z^{2}\right],
$$

where $A, B$ are called cosmic scale factors which are function of time $\mathrm{t}$ and $m$ is an arbitrary constant.

The energy momentum tensor for perfect fluid has of the form

$$
T_{i j}=(\rho+p) u_{i} u_{j}-p g_{i j}
$$

satisfying the equation of state

$$
p=w \rho, 0 \leq w \leq 1,
$$


where $p$ is pressure, $\rho$ is the energy density while $u^{i}=\delta_{4}^{i}$ is the four velocity vector which satisfies

$$
u^{i} u_{i}=1 .
$$

From equation (1), we have the field equations

$$
\begin{aligned}
& -2 \frac{\ddot{B}}{B}+2 \frac{\dot{A} \dot{B}}{A B}+\frac{\dot{A} \dot{F}}{A F}-\frac{\ddot{F}}{F}--\frac{2 m^{2}}{A^{2}}=\frac{k}{F}(\rho+p) . \\
& -\frac{\ddot{A}}{A}-\frac{\ddot{B}}{B}+\frac{\dot{A} \dot{B}}{A B}+\frac{\dot{B}^{2}}{B^{2}}+\frac{\dot{B} \dot{F}}{B F}-\frac{\ddot{F}}{F}-\frac{2 m^{2}}{A^{2}}=\frac{k}{F}(\rho+p) . \\
& \left(\frac{\dot{A}}{A}-\frac{\dot{B}}{B}\right)=0 .
\end{aligned}
$$

On integrating equation (8), we have

$$
A=k B
$$

where $k$ is constant of integration

The average scale factor \& volume scale factor defined as

$$
\begin{aligned}
& a=\left(A B^{2}\right)^{\frac{1}{3}} \\
& V=a^{3}=A B^{2} .
\end{aligned}
$$

We also defined the generalized Hubble parameter $H$ and deceleration parameter $q$ as

$$
\begin{aligned}
& H=\frac{\dot{a}}{a}=\frac{1}{3}\left[H_{1}+H_{2}+H_{3}\right], \\
& q=-\frac{a \ddot{a}}{\dot{a}^{2}}=-\frac{\ddot{a}}{a H^{2}}
\end{aligned}
$$

where $H_{1}=\frac{\dot{A}}{A}, \quad H_{2}=H_{3}=\frac{\dot{B}}{B}$ are the directional Hubble parameters in the direction of $x, y, z$ axes respectively.

The expansion scalar $\theta$ and shear scalar $\sigma^{2}$ are defined as follows

$$
\begin{gathered}
\theta=u_{; i}^{i}=\frac{\dot{A}}{A}+\frac{2 B}{B}, \\
\sigma^{2}=\frac{1}{2} \sigma_{i j} \sigma^{i j}=\frac{1}{3}\left[\frac{\dot{A}}{A}-\frac{\dot{B}}{B}\right]^{2},
\end{gathered}
$$

where $\quad \sigma_{i j}=\frac{1}{2}\left(\nabla_{j} u_{i}+\nabla_{i} u_{j}\right)-\frac{1}{3} g_{i j} \theta$.

The mean anisotropy parameter $A_{m}$ is given by

$$
A_{m}=\frac{1}{3} \sum_{i=1}^{3}\left(\frac{H_{i}-H}{H}\right)^{2} .
$$

After solving (6) and (7), we have 


$$
\frac{A}{B}=d_{1} \exp \left[c_{1} \int \frac{d t}{a^{3} F}\right],
$$

where $d_{1}$ and $c_{1}$ are constants of integration.

Using equation (11) and (18), the metric functions turn out to be

$$
\begin{aligned}
& A=a p_{1} \exp \left[q_{1} \int \frac{d t}{a^{3} F}\right], \\
& B=a p_{2} \exp \left[q_{2} \int \frac{d t}{a^{3} F}\right],
\end{aligned}
$$

where $\quad p_{1}=d_{1}^{\frac{2}{3}}, p_{2}=d_{1}^{\frac{-1}{3}}$,

and

$$
q_{1}=\frac{2}{3} c_{1}, q_{2}=\frac{-1}{3} c_{1}
$$

Satisfying the relations

$$
p_{1} p_{2}^{2}=1, \quad q_{1}+2 q_{2}=0
$$

In order to solve the Einstein's field equations in $f(R)$ theory of gravity, we used the a special form of deceleration parameters which defined by Abdussatter and Prajapati [26], as

$$
q=-\frac{\alpha}{t^{2}}+\beta-1,
$$

where $\alpha>0, \beta>1$ is a constant.

Equation (13) can be integrated to obtain the scale factor as

$$
a(t)=e^{k_{2}} \exp \left[\int \frac{1}{\int(1+q) d t+k_{1}} d t\right] .
$$

where $k_{1}$ and $k_{2}$ are constants of integration.

Using equations (24) and (25), we calculated mean scale factor by substituting $k_{1}=0$ as

$$
a(t)=e^{k_{2}}\left(t^{2}+\frac{\alpha}{\beta}\right)^{\frac{1}{2 \beta}},
$$

Sharif and Shamir [15] have established a result in the context of $f(R)$ gravity which show that

$$
F \propto a^{b} \text {. }
$$

Thus, we have

$$
F=l a^{b},
$$

where $l$ is the constant of proportionality, $b$ is any integer.

We use the value of $a(t)$ for $k_{2}=0, b=-1, \beta=1$ in the equations (19) and (20), we obtained the scale factor as

$$
A=p_{1}\left(t^{2}+\alpha\right)^{1 / 2} \exp \left(\frac{q_{1}}{l \sqrt{\alpha}} \tan ^{-1} \frac{t}{\sqrt{\alpha}}\right)
$$




$$
B=p_{2}\left(t^{2}+\alpha\right)^{1 / 2} \exp \left(\frac{q_{2}}{l \sqrt{\alpha}} \tan ^{-1} \frac{t}{\sqrt{\alpha}}\right)
$$

Hence geometry of the universe (2) is given by

$$
d s^{2}=d t^{2}-\left(t^{2}+\alpha\right)\left\{p_{1}^{2} \exp \left(\frac{2 q_{1}}{l \sqrt{\alpha}} \tan ^{-1} \frac{t}{\sqrt{\alpha}}\right) d x^{2}-e^{2 m x} p_{2}^{2} \exp \left(\frac{2 q_{2}}{l \sqrt{\alpha}} \tan ^{-1} \frac{t}{\sqrt{\alpha}}\right)\left[d y^{2}+d z^{2}\right]\right\}
$$

The volume scale factor is given by

$$
V=\left(t^{2}+\alpha\right)^{3 / 2} \text {. }
$$

The directional Hubble parameters in the directions of $x, y$ and $z$ axis are found to be

$$
\begin{aligned}
& H_{1}=\frac{q_{1}}{l\left(t^{2}+\alpha\right)}+\frac{t}{\left(t^{2}+\alpha\right)} \\
& H_{2}=H_{3}=\frac{q_{2}}{l\left(t^{2}+\alpha\right)}+\frac{t}{\left(t^{2}+\alpha\right)}
\end{aligned}
$$

The mean Hubble parameter becomes

$$
H=\frac{t}{\left(t^{2}+\alpha\right)}
$$

The expansion scalar $\theta$ is given by

$$
\theta=\frac{3 t}{\left(t^{2}+\alpha\right)}
$$

The shear scalar $\sigma^{2}$ calculated as

$$
\sigma^{2}=\frac{\left(q_{1}-q_{2}\right)^{2}}{3 l^{2}\left(t^{2}+\alpha\right)^{2}}
$$

The mean anisotropy parameter $A_{m}$ turns out to be

$$
A_{m}=\frac{2\left(q_{1}-q_{2}\right)^{2}}{9 l^{2} t^{2}}
$$

The energy density and pressure of the universe for stiff matter obtained as

$$
2 k \rho=2 k p=\frac{\left(t^{2}+\alpha\right)^{\frac{1}{2}}}{l}\left[\begin{array}{c}
\frac{\left(q_{1}-6 q_{2}\right) t}{l}+\frac{2 q_{2}\left(q_{2}-q_{1}\right)}{l^{2}}+t^{2}-\alpha \\
-\frac{2 m^{2}\left(t^{2}+\alpha\right)}{p_{1}^{2}} \exp \left(\frac{-2 q_{1}}{l \sqrt{\alpha}} \tan ^{-1} \frac{t}{\sqrt{\alpha}}\right)
\end{array}\right],
$$

However, function of Ricci scalar for Bianchi type-V model is given by

$$
f(R)=\frac{1}{\left(t^{2}+\alpha\right)^{5 / 2}}\left[\begin{array}{l}
\frac{3 m^{2} l\left(t^{2}+\alpha\right)}{p_{1}^{2}} \exp \left(\frac{-2 q_{1}}{l \sqrt{\alpha}} \tan ^{-1} \frac{t}{\sqrt{\alpha}}\right)-\frac{3 l\left(5 t^{2}+3 \alpha\right)}{2}+\frac{q_{1}\left(2 q_{1}+q_{2}\right)}{l} \\
+\frac{\left(t^{2}+\alpha\right)^{3}}{2 l}\left(\frac{\left(q_{1}-6 q_{2}\right) t}{l}+\frac{2 q_{2}\left(q_{2}-q_{1}\right)}{l^{2}}+t^{2}-\alpha\right)
\end{array}\right],
$$


where

$$
R=\frac{2}{\left(t^{2}+\alpha\right)}\left[\frac{q_{2}\left(2 q_{1}+q_{2}\right)}{l^{2}\left(t^{2}+\alpha\right)}+\frac{3 m^{2}}{p_{1}^{2}} \exp \left(\frac{-2 q_{1}}{l \sqrt{\alpha}} \tan ^{-1} \frac{t}{\sqrt{\alpha}}\right)-3\right]
$$

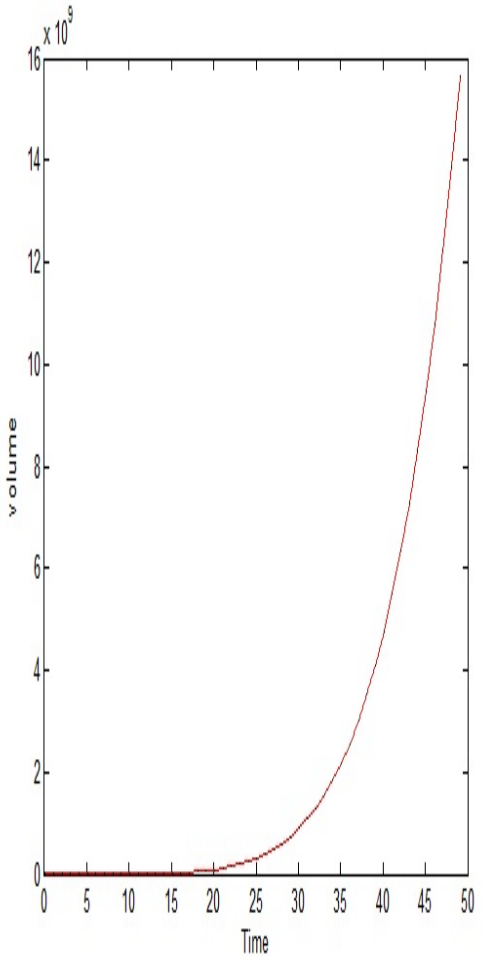

Fig.(1) Volume vs Time

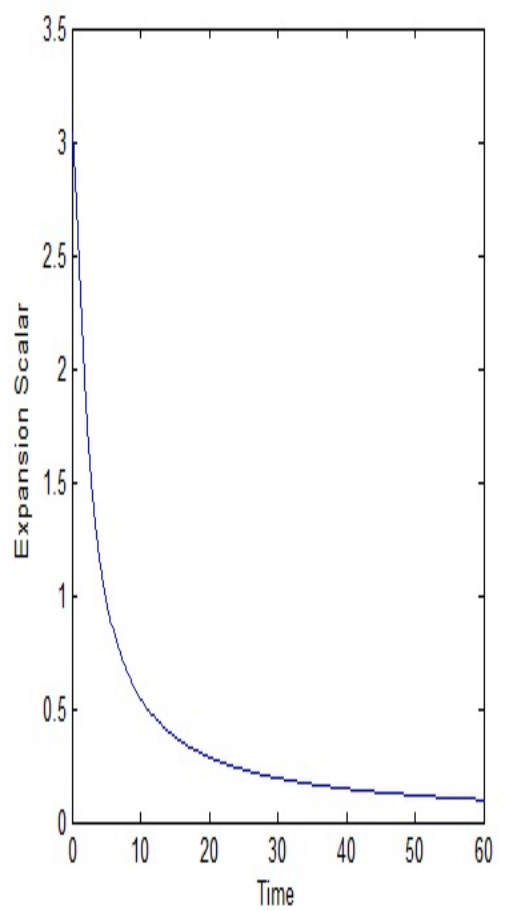

Fig.(2) Expansion Scalar vs Time

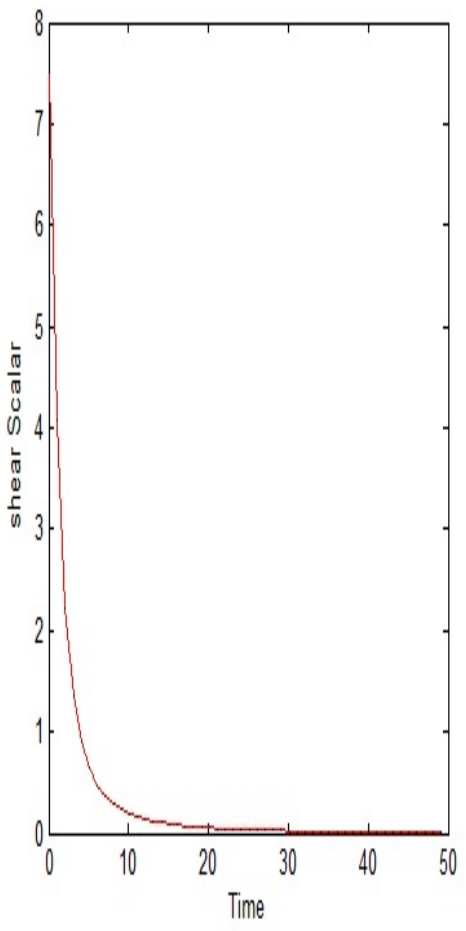

Fig.(3) Shear scalar vs Time

\section{CONCLUding REMARK}

In this paper, we have examined the non-vacuum perfect fluid solution of LRS Bianchi type-V spacetime in the $f(R)$ theory of gravity. We used stiff matter to obtained energy density and pressure of the universe. Also the time dependent deceleration parameter proposed by Abdussatter and Prajapati helped to discuss the physical behavior of the model. The function of the Ricci scalar is also evaluated. The observation as under:

1) The model is free from initial singularity.

2) The spatial volume $\mathrm{V}$ is zero at $t \rightarrow 0$ and increases infinitely with time. This shows that at the initial epoch the universe starts with zero volume and expands uniformly as shown in Fig.(1).

3 ) It is interesting to note that the present model is shear free and isotropic throughout expansion of the universe if $q_{1}=q_{2}$, otherwise model is not shear free and it is an anisotropic.

4) The graph of expansion scalar and shear scalar verses time indicates that shear scalar \& expansion scalar are large as $\mathrm{t}=0$ and become constant when time is increases as shown in Fig.(2) \& Fig.(3) respectively.

\section{REFERENCES}

[1] Perlmutter, S., et al. (1997) Measurement of the cosmological parameters $\Omega$ and $\Lambda$ from the first seven supernovae at $z \geq 0.35$. The Astrophysical Journal, 483, 565. http://dx.doi.org/10.1086/304265

[2] Perlmutter, S., et al. (1998) Discovery of Supernovae Explosion at Half the Age of the Universe. Nature, 391, 51-54. http://dx.doi.org/10.1038/34124

[3] Reiss, A.G., et al. (1998) Observational Evidence from super-novae for an Accelerating Universe and a Cosmological Constant. The Astrophysical Journal, 116, 1009-1038.

[4] Perlmutter, S. et al. (1999) Measurement of and 42 high-Redshift Supernovae. The Astrophysical Journal, 517, 565- 586. http://dx.doi.org/10.1086/307221 
[5] Caldwell, R.R. and Doran, M. (2004) Cosmic Microwave Background and Supernova Constraints on Quintessence: Concordance Regions and Target Models. Physics Review D, 69, 103517. http://dx.doi.org/10.1103/PhysRevD.69.103517

[6] Riess, A.G., et al. (2004) Type Ia Supernova Discoveries at $z>1$ from the Hubble Space Telescope: Evidence for the Past Deceleration and Constraints on Dark Energy Evolution. The Astrophysical Journal, 607, 665-678. http://dx.doi.org/10.1086/383612

[7] Nojiri, S. and Odintsov, S.D.: Problems of Modern Theoretical Physics,A Volume in honour of Prof. Buchbinder, I.L. in the occasion of his 60thbirthday, p.266-285, (TSPU Publishing, Tomsk), arXiv:0807.0685.

[8] Nojiri, S. and Odintsov, S.D. : Introduction to modified gravity and gravitational alternative for dark energy, Int. J. Geom. Meth. Mod.Phys. 115(2007)4.

[9] M. Sharif and H. RizwanaKausar: Anisotropic Fluid and Bianchi Type III Model in $f(R)$ Gravity, arXiV:1101.3372 v1[gr-qc] 18 Jan 2011.

[10] M. Sharif et. al.: Energy distribution in $f(R)$ gravity, arXiV:0912.3532 v1 [gr-qc] 18 Dec 2009.

[11] K..S.Adhav: Bianchi Type III string cosmological Model in $f(R)$ Gravity, Bulg.J.Phys.39(2012)197-2006.

[12] Hollenstein, L. and. Lobo, F.S.N : Exact solutions of $f(R)$ gravity coupled to nonlinear electrodynamics, Phys. Rev. D78(2008)124007.

[13] Kumar, S. and Singh, C.P.: Astrophys. Space Sci. 312(2007)57.

[14] M. Sharif and H. Rizwana Kausar: Non-vacuum Solutions of Bianchi Type VIo Universe in $f(R)$ Gravity, arXiV:1010.2554 v1[gr-qc] 13 Oct 2010.

[15] M. Sharif and M. Farasat Shamir: Non-Vacuum Bianchi Types-I and $V$ in $f(R)$ Gravity, arXiV: 100502798 v1 [gr-qc] may 2010.

[16] M. Sharif and H. R. Kausar: Phys. Lett. B 697 (2011) 1.

[17] M. Sharif and W. Saira: Eur. Phys. J. C 72 (2012) 1876; C. Aktas, S. Aygun and I. Yilmaz: Phys. Lett. B 707 (2012) 237.

[18] S. Capozziello: Phys. Lett. B 639 (2012) 135; S. Capozziello and M. Laurentis: Phys. Rep. 509 (2011) 1; D. Yunshuang, Z. Hongsheng and L.Xin-Zhou: Eur. Phys. J. C 71 (2011) 1660; Y. Bisabr: Phys. Lett. B 690 (2011) 456; V. Faraoni: Class. Quantum Grav. 26 (2009) 145014.

[19] M. Farasat Shamir et.al.: "Exact Solutions of Bianchi Types I and V Space-times in $f(R, T)$ Theory of Gravity, arXiv:1207.0708 v1 [gr-qc] 2 July2012.

[20] R.L.Naidu et.al , Bianchi Types- $V$ bulk viscous string cosmological model in $f(R, T)$ gravity, Astrophys. Space Sci. DOI 10.1007/s10509-013-1540-0(2013).

[21] H.R. Ghate et.al.: Bianchi Types-IX viscous string cosmological model models in $f(R, T)$ gravity with special form of declaration parameter, International Journal of Theoretical and Mathematical Physics, 2014,4(6),240-247.

[22] P.K. Sahoo et.al.,:Kaluza-Klein cosmological model in $f(R, T)$ gravity, arXiv:1011.4735 v2 [gr-qc] 24 Nov2014

[23] Banerjee and Sanyal : Gen relativity Gravit., 20,103 (1988).

[24] Collins: Comm. Maths. Phys., 39, 131 (1974).

[25] Wainwrig: Gen. Relativ. Gravit. 10,259 (1979).

[26] Abdussattar S.R. Prajapati.: Role of deceleration parameter and interacting dark energy in sinularity avoidance, Astrophys Space Sci (2011) 331: 657-66 